\title{
Fault-tolerant attitude control for flexible spacecraft with input saturation
}

\begin{abstract}
Fault-tolerant constrained attitude controllers are proposed for flexible spacecraft in the presence of input saturation and actuator fault, as well as model uncertainty and external disturbance. Two input saturations, that is amplitude saturation and, amplitude and rate saturation are considered and simple and effective compensators are designed to deal with the effect of input saturation. Two parameter update laws are designed to endure stuck fault, partial and complete loss of effectiveness fault. The stability of the resulting closedloop systems by the proposed controllers is guaranteed by Lyapunov-based approach. The effectiveness of the proposed algorithms is assessed through numerical simulations.
\end{abstract}

Keywords: fault-tolerant, attitude control, flexible spacecraft, input saturation
Volume 2 Issue 4 - 2017

\author{
Haihui Long, Jiankang Zhao \\ School of Electronic Information and Electrical Engineering, \\ Shang Hai Jiao Tong University, China
}

Correspondence: Jiankang Zhao, School of Electronic Information and Electrical Engineering, Shang Hai Jiao Tong University, China, Tel +8618801969342

Email zhaojiankang@sjtu.edu.cn

Received: April 24, 2017 | Published: June 12, 2017

\section{Introduction}

In the past several decades, the attitude control problem of spacecraft has attracted a great deal of attention due to its important applications. ${ }^{1-3}$ As actuation devices generate toques with only limited amplitude and/or rate, ${ }^{4-6}$ input saturation may frequently occur during the entire attitude maneuvers of a spacecraft. As discussed in ${ }^{7,8}$ input saturation can severely degrade closed-loop system performance or even in some cases cause system instability if they are not carefully tackled in attitude control process. Because of this, many researchers have focused on input saturation in attitude controller design of spacecraft. ${ }^{9-13}$ In $\mathrm{Hu},{ }^{12}$ neural network was used to estimate the unknown input saturation and then the effect of input saturation was compensated by inserting the saturation compensator into the feed-forward loop of the system. In $\mathrm{Hu},{ }^{13}$ an auxiliary variable was employed to compensate the effect of input saturation in attitude controller design. However, the tracking error would be modified to get a stable control system with this auxiliary variable. In addition, during the spacecraft mission, actuators may undergo faults due to aging or accidents, such as partial loss of effectiveness, stuck and outage. These faults may cause system instability or even end up with catastrophic events if they are not well handled. Therefore, designing a controller that is robust to these actuator faults is one of the significant issues that need to be considered by researchers. Fault-tolerant control (FTC $)^{14,15}$ is considered as one of the most effective approaches for maintaining stability and expected performance of system during the actuator faults occurrence. More and more literatures have focused on fault-tolerant attitude control for a spacecraft; see. ${ }^{16-22}$ In Jiang, ${ }^{21}$ the authors proposed fault-tolerant attitude control of spacecraft to accommodate the partial loss of effectiveness faults in actuators with a prior knowledge of the lower bound of the effectiveness factor. In $\mathrm{Hu},{ }^{22}$ the knowledge of the bound of the partial effectiveness factor was not need by employing an update law to estimate the lower bound online. However, these mentioned literatures above less considered another important fault scenario of actuators, i.e., stuck fault. As far as we know, in attitude control design, there are few literatures that take stuck fault into account explicitly expect for $\mathrm{Hu} .{ }^{23} \mathrm{But}$ in $\mathrm{Hu},{ }^{23}$ input saturation were not considered, which might be sometimes conservative in practical applications.

Model uncertainty and external disturbance are another two large challenges that need to be considered in the attitude controller design of a spacecraft. Inverse optimal method is an attractive control approach for system with model uncertainty and external disturbance as it has the properties of robust to uncertainty and disturbance, and can achieve $H$ optimality without the need to solve the HamiltonJacobi-Isaacs partial differential (HJIPD) equation directly. Several researchers studied this approach to solve the attitude control problem of spacecraft. ${ }^{24-26}$ However, these literatures above more or less ignored the constraints on control input and the scenario of actuator faults. In addition, the vibration effect of the flexible appendages induced by the orbiting attitude slewing operation was also not handled explicitly. Therefore when input saturation and actuator faults occur simultaneously, stability will no long be guaranteed by using these existing inverse optimal methods. Furthermore, the stability of system becomes worse when serious vibration effect of the flexible appendages happens, because it tends to be aggressive while seeking the expected control performance. To overcome the shortcomings of the preceding research for spacecraft attitude control systems, novel constrained fault-tolerant attitude control strategies are proposed in this work for flexible spacecraft in the presence of actuator faults, input saturation, uncertainty inertia matrix and external disturbance. The main contributions of this paper are shown as follows:

a. Unlike existing compensator in $\mathrm{Hu},{ }^{13}$ in our work, a simple and effective compensator is introduced and embedded to the feedback controllers to eliminate the effect of input saturation.

b. To handle the stuck faults and loss of effectiveness (including partial and complete loss of effectiveness) in actuator for a spacecraft, a new Lemma is first proposed and rigorous proof is presented. Two robust constrained fault-tolerant controllers, accounting for actuator amplitude constraint and, actuator amplitude and rate constraints, respectively, are proposed by introducing two parameter update laws to estimate the unknown parameters caused by actuator faults.

c. In comparison with the existing literatures ${ }^{24-26}$ we extend the inverse optimal method to flexible spacecraft with input saturation and actuator faults.

The remainder of the paper is organized as follows: Section 2 presents the mathematical model of flexible spacecraft and control problem. Robust constrained fault-tolerant attitude controllers are derived in Section 3. Numerical simulation results of a flexible spacecraft with the derived controllers are given in Section 4, and Section 5 comprises conclusions and possibilities of future work. 


\section{Model description and problem formulation}

This section briefly introduces the attitude kinematics and dynamic models of a flexible spacecraft. The model of the actuator faults is also established. The attitude kinematic equation of spacecraft can be expressed by unit quaternion as

$$
\dot{Q}=\left[\begin{array}{c}
\dot{q}_{0} \\
\dot{q}
\end{array}\right]=\frac{1}{2}\left[\begin{array}{c}
-q^{T} \\
q^{x}+q_{0} I
\end{array}\right] \omega
$$

Where $\omega \in R^{3}$ is the angular velocity of the spacecraft with respect to an inertial frame and expressed in body frame, Q is the unit quaternion with the unit norm constraint $\mathrm{Q},\|Q\|=q_{0}^{2}+q^{T} q=1$ given by

$$
Q=\left[\begin{array}{c}
q_{0} \\
q
\end{array}\right]=\left[\begin{array}{c}
\cos (\theta / 2) \\
e \sin (\theta / 2)
\end{array}\right]
$$

$q=\left[q_{1}, q_{2} q_{3}\right]^{T} \in R^{3}$ and $q_{0} \in R^{1}$ are the vector part and the scalar part of the unit quaternion $\mathrm{Q}$, respectively, $e \in R^{3} 3$ and $\theta$ denote the Euler axis and Euler angle, respectively, I is the identity matrix with appropriate dimension, and the superscript $\mathrm{X}$ is an operator which is denoted by

$$
a^{x}=\left[\begin{array}{ccc}
0 & -a_{3} & a_{2} \\
a_{3} & 0 & -a_{1} \\
-a_{2} & a_{1} & 0
\end{array}\right]
$$

$a$ is a three dimensional vector with elements $a_{1}, a_{2}$ and $a_{3}$. Obviously, $a^{x}$ is a skew-symmetric matrix.

The dynamic equations of a flexible spacecraft can be written as ${ }^{12}$

$$
\begin{gathered}
J_{s} \dot{\omega}+\sigma^{T} \ddot{\eta}=-\omega^{x}\left(J_{S} \omega+\sigma^{T} \dot{\eta}\right)+u+d_{s} \\
\ddot{\eta}=-D \dot{\eta}-E \eta-\sigma \dot{\omega}
\end{gathered}
$$

whereJs $J_{s} \in R^{3 \times 3}$ represents the inertia matrix of the whole spacecraft, $\quad \eta \in R^{N}$ denotes the model coordinate vector with is the model number, $\sigma \in R^{N \times 3}$ denotes the coupling matrix between the elastic and rigid structure, $u \in R^{3} \mathrm{~N}$ is the actual control torque, $d_{s} \in R^{3}$ is disturbance torques, $D=\operatorname{diag}\left\{2 \xi_{i} \vartheta_{i}^{\frac{1}{2}}, i=1,2, \ldots, N\right\}$ and $E=\operatorname{diag}\left\{\vartheta_{i}, i=1,2, \ldots, N\right\}$ are the damping and stiffness matrices, $\underline{1}$ respectively, $\xi_{i}$ is the corresponding damping ratio, and $\vartheta_{i}^{2}$ is the natural frequency. When considering actuator faults, the actual control torque $\mathrm{u}$ can be modeled as

$$
u(t)=\sum\left(\wedge u_{k h}(t)+(I-\wedge) F(t) u_{c}(t)\right)
$$

Where $\sum \in R^{3 \times L}, L \geq 3$ is the actuators distribution matrix, $\wedge=\operatorname{diag}\left(\wedge_{1}, \ldots, \wedge_{L}\right)$ with $\wedge \in\{0,1\}$ being the fault switch factor, $u_{k h}(t)=\left[u_{k h 1}(t), u_{k h 2}(t), \ldots, u_{L}(t)\right]^{T} \in R^{L}$ is the struck fault of actuators, $u_{c}=\left[u_{c 1}, u_{c 2}, u_{c l}\right]^{T} \in R^{L}$ denotes the input torque of actuators, $F(t)=\operatorname{diag}\left(F_{1}(t) F_{2}(t), \ldots, F_{L}(t)\right)$ represents effectiveness matrix of actuators. $\mathrm{F}(\mathrm{t})$ can be modeled as ${ }^{19}$

$F_{i}=A_{F_{i}}+\frac{B_{F_{i}}}{2}\left(\cos \left(\vartheta_{F_{i}}\right)-1\right), A_{F_{i}} \in[0,1], B_{F_{i}} \in[0,1], i=1,2, \ldots ., \mathrm{L}$

Where $A_{F_{i}}$ denotes the magnitude of the actuator effectiveness and, $B_{F_{i}}$ and $\omega_{F_{i}}$ are the amplitude and frequency of the high-frequency component, respectively, with $A_{F_{i}} \geq B_{F_{i}}$. Therefore, we have $F_{i} \geq 0$, $\mathrm{i}=1,2, \ldots, \mathrm{L}$.

Remark 1: Note that $u_{k h i}$ and $F_{i}, \mathrm{i}=1, \ldots, \mathrm{L}$, can be constant or time-varying. From (6) we can conclude that, for the $\mathrm{i}$ th actuator, in the case of $\wedge_{i}=1$ and $u_{k h i} \neq 0$, the actuator occurs stuck fault; In the case of $\wedge_{i}=0$ and $0<F_{i}<1$, the actuator experiences partial loss of actuator effectiveness; In the case of $\wedge_{i}=0, F_{i}=0$ or $\wedge_{i}=1$, $u_{k h i}=0$, the actuator undergoes outage; In the case of $\wedge_{i}=0, F_{i}$ $=1$, the actuator is fault-free. If all the actuators are fault-free in the whole attitude control process, the dynamic system (4) become the nominal system where $u=\sum u_{c}$. In the following controller designs, the fault system and nominal system are considered, respectively.

In practice, due to fuel consumption, out-gassing during operation, onboard payload motion and rotation of flexible appendages and so on, the inertial matrix $\mathrm{J}_{\mathrm{s}}$ of the whole spacecraft structure is time varying and uncertain. We divide it into two parts, that $J_{s}=J+\Delta J$, where $\mathrm{J}$ and $\Delta J$ represent the nominal value component and the parameter perturbation component of the inertial matrix $J_{s}$, respectively. As $\Delta J$ is time-varying and unknown, the terms $\Delta J \dot{\omega}$ and $\omega \times \Delta J \omega$ are also time-varying and unknown. Therefore we can treat them as the disturbance to be tackled in the controller design. When considering $\Delta J \dot{\omega}, \omega \times \Delta J \omega$ and $d_{s}$ as the total disturbance $\mathrm{d},(4)$ can be rewritten as

$$
J \dot{\omega}+\sigma^{T} \ddot{\eta}=-\omega \times\left(J \omega+\sigma^{T} \dot{\eta}\right)+u+d
$$$$
\text { Where } d(t)=\Delta J \dot{\omega}-\omega \times \Delta J \omega+d_{s}(t) \text {. }
$$

The following assumptions are taken throughout this paper.

Assumption 1: Both $\mathrm{J}$ and $J-\sigma^{T} \sigma$ are known positive definite symmetric and bounded constant matrices.

Assumption 2: The actuators distribution matrix $\sum$, fault switch matrix $\wedge$ and actuator effectiveness matrix $F$ satisfy $\operatorname{rank}(\Sigma)=\operatorname{rank}(\Sigma(I-\wedge) F)=3$.

Assumption 3: The stuck faults $u_{k h i}, i=1, \ldots, L$ are bounded, i.e., $\left|u_{k h i}\right| \leq \bar{u}_{k h}$.

Assumption 4: The total disturbance $d$ is bounded with a bound constant $\bar{\varepsilon}$, i.e., $\int_{0}^{t}\|d(s)\|^{2} d s<\bar{\varepsilon}$ for $\forall t \geq 0$, where $\|\bullet\|$ denotes the norm of vector or matrix. 
Assumption 5: The control input torque of actuators $u_{c}(t)=\left[u_{c 1}(t), u_{c 2}(t), u_{c 3}(t)\right]^{T}$ satisfies

$$
\left\|\sum u_{c}\right\| \leq \Gamma_{\max }
$$

Where $\Gamma_{\max }>0$ max is a known constant.

Remark 2: Assumption 2 is used to guarantee that the remaining actuators can still achieve the designed control aims even though some actuators undergo outage or stuck. From Assumption 2 and the $\operatorname{fact} \min \{\operatorname{rank}(\Sigma), \operatorname{rank}((I-\wedge) F)\} \geq \operatorname{rank}\left(\sum(I-\wedge) F\right)=3$ , we have $\operatorname{rank}((I-\wedge) F) \geq 3$. From Assumption 5, we have c $\|u\|=\left\|\sum u_{c}\right\| \leq \Gamma_{\text {max }}$ when all actuators are fault-free. The rest-to-rest maneuver of the spacecraft is considered in this work. The control aim is to design a controller $\mathrm{c} u$ for the system in (1), (4)-(6) such that for all initial conditions the desired rotations are achieved in the presence of model uncertainty, external disturbances, inputs saturation or ever actuator faults under Assumptions 1-5, that is

$$
\lim _{t \rightarrow \infty} q(t)=0, \lim _{t \rightarrow \infty} \omega(t)=0, \lim _{t \rightarrow \infty} \eta(t)=0, \lim _{t \rightarrow \infty} \dot{\eta}(t)=0
$$

\section{Robust attitude control for flexible spacecraft}

\section{Mathematical preliminaries}

This section includes some important mathematical preliminaries required for the rest of the paper. Consider the nonlinear dynamic system

$$
\dot{x}=f(x)+g_{1}(x) d+g_{2}(x) u
$$

Where $x \in R^{n}$ is the state, $u \in R^{p}$ is the input, $d \in R^{s}$ is the disturbance, and $g_{2}: R^{n} \rightarrow R^{n \times p}$ are smooth vector- or matrixvalued function, respectively. System (9) is said to be input-to-state stable (ISS) $)^{27,28}$ from d to $\mathrm{x}$ if the following property is satisfied:

$$
|x(t)| \leq \alpha(|x(0)|, t)+\rho\left(\sup _{0 \leq \tau \leq t}|d(\tau)|\right)
$$

Where $\alpha$ is a class $\kappa$ L functions and $\rho$ is a class $\kappa$ function.

Definition 1: A smooth function positive definite and radically unbounded $V(x): R^{n} \times R_{\perp} \rightarrow R_{\perp}$ is called an input-to-state stabilizable control Lyapunov function (ISS-CLF) for (9) if there exists some class $\kappa$ function $\rho$ such that the following implication holds for all $\mathrm{x} \neq 0$ and all $\mathrm{d}^{27,28}$

$$
|x| \geq \rho(|d|) \Rightarrow \inf _{u} \dot{V} \leq 0
$$

Definition $2^{27}$ : For system (9), let V is an ISS-CLF with the control law $u=\frac{1}{2} \xi^{\bullet}(x)=-F(x)^{-1}\left(L_{g 2} V\right)^{T}$, where $F(x)$ is positive definite symmetric matrix and $L_{g 2} \mathrm{~V}$ is Lie derivative defined as $L_{g 2} V=(\partial V(x) / \partial x) g 2(x)$, then the stabilizing control law

$$
u=\xi^{\bullet}(x)=-2 F(x)^{-1}\left(L_{g 2} V\right)^{T}
$$

Is $H_{\infty}$ inverse optimal with respect to the cost functional

$$
J(u)=\sup _{d \in D}\left\{\lim _{t \rightarrow \infty}\left[4 V(x(t), t)+\int_{0}^{t}\left(l(x, t)+u^{T} F(x) u-\gamma^{2}\|d\|^{2}\right) d \kappa\right]\right\}
$$

Where

$l(x)=-4 L_{f} V-\frac{4}{\gamma^{2}} L_{g_{1}} V\left(L_{g_{1}} V\right)^{T}+4 L_{g_{2}} V F^{-1}\left(L_{g_{2}} V\right)^{T}>0, \forall x \neq 0, \mathrm{D}$ is the set of locally bounded functions of $\mathrm{x}$ and $\gamma>0$.

Remark 3: A necessary and sufficient condition for system being ISS is the existence of an ISS-CLF [28]. The main characteristic of the inverse optimal approach is that the meaningful cost function is a posteriori determined from the stabilizing feedback control law. The inverse optimal controller $u=\xi^{*}(x)$ (x) in (11) can achieve $\gamma$ level of $H_{\infty}$ disturbance attenuation for all $t \geq 0$. Compared with nonlinear $H$ control, the inverse optimal method solves the nonlinear optimalassignment problem with respect to a meaningful cost functional without solving the HJIPD equation explicitly.

\section{Robust controller design for flexible spacecraft}

For simplicity, we denote the following variable $\mathrm{v}$ by $v=\sigma \omega+\dot{\eta}$ differentiating the variable v yields $v=\sigma \omega+\dot{\eta}=-D v-E \eta+D \sigma \omega$. Let $\xi=\left[\eta^{T}, v^{T}\right]^{T}$, and then (5) can be written as

$$
\dot{\xi}=\left[\begin{array}{cc}
0 & I \\
-E & -D
\end{array}\right] \xi+B \omega
$$

Where $\mathrm{B}=\left[\begin{array}{c}-\sigma \\ D \sigma\end{array}\right]$. Since matrix $\left[\begin{array}{cc}0 & I \\ -E & -D\end{array}\right]$ has all its eigen values in the left-hand plane, there exists a symmetric and positivedefinite solution $P \in R^{2 N \times 2 N}$ satisfying

$$
P\left[\begin{array}{cc}
0 & I \\
-E & -D
\end{array}\right]+\left[\begin{array}{cc}
0 & I \\
-E & -D
\end{array}\right]^{T} P=-T
$$

Where $T \in R^{2 N \times 2 N}$ is positive definite symmetric matrix In view of (7), one has

$\left(J-\sigma^{T} \sigma\right) \dot{\omega}=-\omega^{\times}\left(J-\sigma^{T} \sigma\right)-\sigma^{T} D \sigma \omega+\left[\begin{array}{ll}\sigma^{T} E & \sigma^{T} D-\omega^{\times} \sigma^{T}\end{array}\right] \xi+u+d$

Define $x=\omega+K q$

Where $K \in R^{3 \times 3}$ and $K=K^{T}>0$. as a result, the subsystem (14) becomes

$$
\dot{x}=\left(J-\sigma^{T} \sigma\right)^{-1}\left[-\omega^{x}\left(J-\sigma^{T} \sigma\right) \mid \omega-\sigma^{T} D \sigma \omega+\frac{1}{2}\left(J-\sigma^{T} \sigma\right) K\left(q_{0} I_{3}+q^{x}\right) \omega+\left[\begin{array}{cc}
\sigma^{T} E & \left.\left.\sigma^{T} D-\omega^{x} \sigma^{T}\right] \xi+u+d\right]
\end{array}\right.\right.
$$

Amplitude constrained attitude controller design: In this section, one controller is presented by constructing control Lyapunov function and using inverse optimal method for normal system in (1) and (4)-(6) with actuator fault-free in the presence of inertia matrix uncertainty, external disturbance and amplitude constraint of actuator. A compensator is proposed and embedded into the feedback controller to eliminate the effect of input saturation. And then a faulttolerant version is designed to deal with the stuck faults and loss of 
effectiveness of actuators by employing two parameter update laws with e-modifications to estimate the unknown parameters caused by actuator faults. For convenience, we firstly define the two saturation functions as

$$
\operatorname{SAT}_{M}\left(\frac{\tau}{2}, \frac{\tau_{\max }}{2}, x\right)=\left\{\begin{array}{cl}
\frac{\tau_{\max } \frac{x}{2}\|x\|}{\|x\|} & \|\tau\| \tau_{\max } \\
\frac{\tau}{2} & \|\tau\| \leq \tau_{\max }
\end{array}\right.
$$

Where $\tau_{\max }>0, x=\left[x_{1}, x_{2}, x_{3}\right]^{T}$ and $\tau=\left[\tau_{1}, \tau_{2}, \tau_{3}\right]^{T} \in R^{3}$, and

$$
\operatorname{SAT}_{R}(h)=\left[c_{1}, c_{2}, c_{3}\right]^{T}
$$

Where $c_{i}=\left\{\begin{array}{ll}R_{i} & \left|h_{i}\right|>R_{i} \\ h_{i} & \left|h_{i}\right| \leq R_{i}\end{array}, i=1,2,3\right.$,

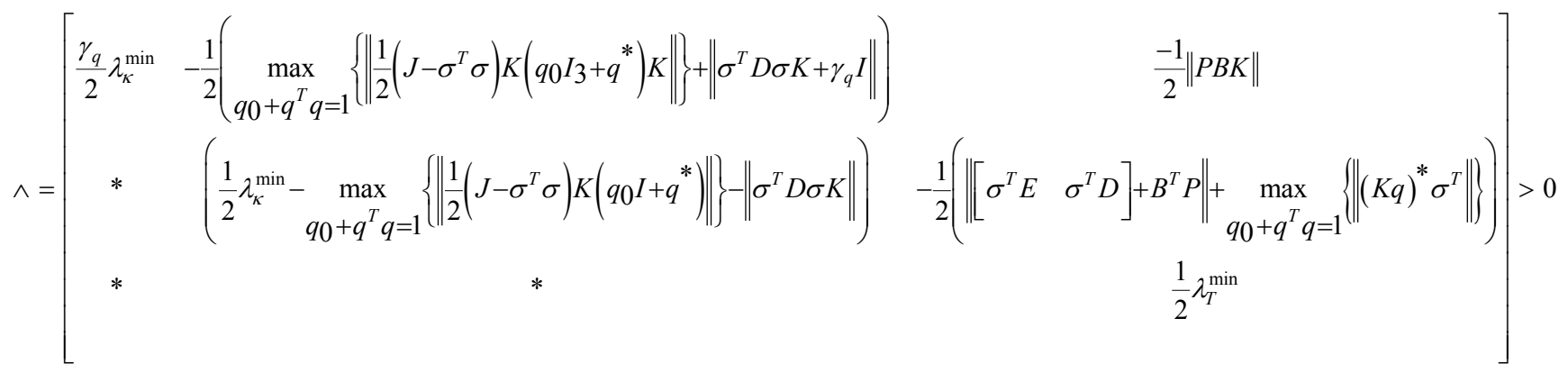

Renders the resulting closed loop system in (1), (12) and (16) ISS

Where $\lambda_{A}^{\text {min }}$ denotes the minimum singular value of matrix A, from the total disturbance d to the state $\left[q^{T}, x^{T}, \xi^{T}\right]^{T}$ furthermore, the then the dynamic feedback control law

$\psi=-\left[\frac{1}{\gamma_{q}}\left\|\omega^{\times}\left(J-\sigma^{T} \sigma\right)^{T}\left(K+\gamma_{q} K_{1}^{-1}\right)\left(\omega^{\times}\left(J-\sigma^{T} \sigma\right)\right)\right\|+2\left\|K_{1}\right\|+\frac{2}{\gamma^{2}}\right] x$
With $h=\left[h_{1}, \mathrm{~h}_{2}, \mathrm{~h}_{3}\right]^{T} \in R^{3}$ and $R=\left[R_{1}, R_{2}, R_{3}\right]^{T} \in R^{3}, R>0$, $=1,2,3$,

In the follows, in order to propose the fault-tolerant constrained controllers, a significant Lemma is proposed.

Theorem 1: For flexible spacecraft system in (1), (12) and (16), under Assumptions 1-5, given $\gamma>0$ , let $\gamma_{q}>0, \quad \kappa=\operatorname{diag}\left(\kappa_{1}, \kappa_{2}, \kappa_{3}\right) \in R^{3 \times 3}, \quad$ where $\kappa_{i}>0, i=1,2,3, \kappa \in R^{3 \times 3}, \kappa_{1} \in R^{3 \times 3}$ and $\kappa_{1} \in R^{3 \times 3}$ be positive definite symmetric matrices. If $\gamma_{q}, \mathrm{~K}$ and satisfy the following inequality

$$
u=-F_{1}^{-1} x=S_{M}\left(\frac{\tau}{2}, \frac{\tau_{\max }}{2}, x\right)
$$

Where $\tau=\psi+2 l$

$$
\begin{aligned}
& l=-\kappa G-\dot{G} \\
& \dot{G}=-\kappa G+\left(u-\frac{\tau}{2}\right)
\end{aligned}
$$

control law $u=-F_{1}^{-1} x=2 \operatorname{SAT}_{M}\left(\frac{\tau}{2}, \frac{\tau_{\max }}{2}, x\right)$

Where $\tau=\psi+l$

$$
\begin{aligned}
& l=-\kappa G-\dot{G} \\
& \dot{G}=-\kappa G+(u-\tau)
\end{aligned}
$$

, and $\psi$ is defined in (22), is inverse optimal in the sense that it minimizes the meaningful functional

$$
J(u)=\lim _{t \rightarrow \infty}\left[4 V(t)+\int_{0}^{t}\left(X(x)+u^{T} F_{1} u-\gamma^{2}\|d\|^{2}\right) d s\right]
$$

$$
\begin{aligned}
& X(x)=-4\left\{\gamma_{q} q^{T} \omega+x^{T}\left[-\omega^{\times}\left(J-\sigma^{T} \sigma\right) \omega-\sigma^{T} D \sigma \omega+\frac{1}{2}\left(J-\sigma^{T} \sigma\right) K\left(q_{0} I+q^{\times}\right) \omega+\left[\begin{array}{cc}
\sigma^{T} E & \sigma^{T} D-\omega^{\times} \sigma^{T}
\end{array}\right] \xi\right]+\xi^{T} P\left(\left[\begin{array}{cc}
0 & I \\
-E & -D
\end{array}\right] \xi+B \omega\right)\right\} \\
& +4 x^{T} F_{1}^{-1} x-\frac{4}{\gamma^{2}} x^{T} x
\end{aligned}
$$


Proof: Consider the smooth positive-definite radically unbounded function

$$
V=\frac{1}{2} \gamma_{q} q^{T} q+\frac{1}{2} \gamma_{q}\left(1-q_{0}\right)^{2}+\frac{1}{2} x^{T}\left(J-\sigma^{T} \sigma\right) x+\frac{1}{2} \xi^{T} P \xi
$$

The time derivate $\mathrm{V}$ along (1), (12) and (16), substituting (21),
(23)-(24) into (20) and based on the definition $\operatorname{SAT}_{M}\left(\frac{\tau}{2}, \frac{\tau_{\max }}{2}, x\right)$ in

(17), using (13), (19)-(21), (22), (31) and the fact $x^{T}\left[0-x^{\times} \sigma^{T}\right] \xi=0$ , we have

$$
\begin{aligned}
& \dot{V}=-\gamma q^{q^{T} K q+\gamma} q^{x^{T}} q+x^{T}\left[-\omega^{\times}\left(J-\sigma^{T} \sigma\right) \omega-\sigma^{T} D \sigma \omega+\frac{1}{2}\left(J-\sigma^{T} \sigma\right) K\left(q_{0} I+q^{\times}\right) \omega+\left[\begin{array}{cc}
\sigma^{T} E & \left.\sigma^{T} D-\omega^{\times} \sigma^{T}\right] \xi+u+d
\end{array}\right]\right. \\
& +\xi^{T} P\left(\left[\begin{array}{cc}
0 & I \\
-E & -D
\end{array}\right] \xi+B \omega\right)
\end{aligned}
$$

$-\gamma_{q} q^{T} K q-\frac{1}{2} \xi^{T} T \xi-x^{T}\left[\frac{1}{2}\left(J-\sigma^{T} \sigma\right) K\left(q_{0} I_{3}+q^{\times}\right) K-\sigma^{T} D \sigma K-\gamma_{q} I\right] q$

$+x^{T} \omega^{\times}\left(J-\sigma^{T} \sigma\right) K q+x^{T}\left[\frac{1}{2}\left(J-\sigma^{T} \sigma\right) K\left(q_{0} I_{3}+q^{\times}\right)-\sigma^{T} D \sigma\right] x$

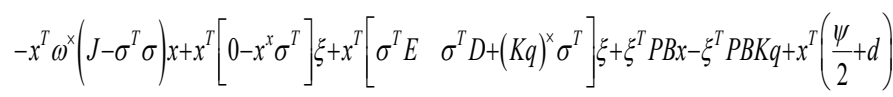

$-\frac{\gamma_{q}}{2} \lambda_{k}^{\min }\|q\|^{2}-\frac{1}{2} \lambda_{T}^{\min }\|\xi\|^{2}-\left(\frac{1}{2} \lambda_{k i}^{\min }-\left\|\frac{1}{2}\left(J-\sigma^{T} \sigma\right) K\left(q_{0} I_{3}+q^{\times}\right)\right\|-\left\|\sigma^{T} D \sigma\right\|\right)\|x\|^{2}$

$-\frac{1}{2}\left\|\sqrt{\gamma_{q}} K_{2}^{\frac{1}{2}} q-\frac{1}{\sqrt{\gamma_{q}}} K_{2}^{\frac{1}{2}}\left(\omega^{\times}\left(J-\sigma^{T} \sigma\right)\right) x\right\|^{2}-\frac{1}{2} x^{T}\left(K_{1}+\left(\omega^{\times}\left(J-\sigma^{T} \sigma\right)\right)\right)^{T}$

$K_{1}^{-1}\left(K_{1+}\left(\omega^{\times}\left(J-\sigma^{T} \sigma\right)\right)\right) x+\left(\left\|\frac{1}{2}\left(J-\sigma^{T} \sigma\right) K\left(q_{0} I_{3}+q^{\times}\right) K\right\|+\left\|\sigma^{T} D \sigma+\gamma_{q} I\right\|\right)\|x\|\|q\|$

$+\|P B K\|\|\xi\| q\left\|+\left(\left\|\left[\begin{array}{cc}\sigma^{T} E & \sigma^{T} D\end{array}\right]+B^{T} P\right\|+\left\|(K q)^{\times} \sigma^{T}\right\|\right)\right\| x\|\xi\| \|$

$\|x\|\left(\|d\|-\|x\| / \gamma^{2}\right) \leq-\lambda_{\wedge}^{\min }\left(\|q\|^{2}+\|x\|^{2}\|\xi\|^{2}\right)+\|x\|\left(\|d\|-\|x\| / \gamma^{2}\right)$

Choosing $\rho(|d|)=\gamma^{2}\|d\| \in \kappa_{\infty}$, then when $\|x\| \geq \gamma^{2}\|d\|$ we have $\dot{V} \leq 0$, which implies that $\mathrm{V}$ in (31) is ISS-CLF for system in (1), (12) and (16) based on Definition 1.Therefore the resulting closed loop system in (1), (12) and (16) is ISS from the total disturbance $\mathrm{d}$ to the state $\left[q^{T}, x^{T}, \xi^{T}\right]$ based on Remark 3. In addition, from the definition of $\chi(x)$ in (30) and the proof above, it is not difficult to yield

$$
\chi(x) \geq 4 \lambda_{\wedge}^{\min }\left(\|q\|^{2}+\|x\|^{2}+\|\xi\|^{2}\right)>0
$$

Which implies that $\chi(x)$ is positive definite. From Definition 2 we get that control law $\mathrm{u}$ in (25) is $H$ inverse optimal control with respect to $(u)$. To this end, from the controller in (25) and the definition in (17), the inequality $\|u\| \leq\left\|\frac{\tau_{\max } x}{\|x\|}\right\| \leq \tau_{\max }$ can be obtained. The proof of Theorem 1 is completed. From (28) we have

$$
\begin{aligned}
& \frac{d}{d t}\left(0.5\|G\|^{2}\right)=G^{T}(\kappa G+((u-\tau))) \\
& \leq-2 \lambda_{\kappa}^{\min }\|0.5 G\|^{2}+\sqrt{2}\|u-\tau \mid\| \sqrt{0.5} G \|
\end{aligned}
$$

Solving the above inequality, we can obtain $\|G(t)\| \leq e^{-\lambda_{\kappa}^{\min } t}\|G(0)\|+\sqrt{2} \max _{0 \leq s \leq t}\|u(s)-\tau(s)\|\left(1-e^{-\lambda_{\kappa}^{\min } t}\right) / 2 \lambda_{\kappa}^{\min }$ , which implies that system in (28) is ISS with input $(u(t)-\tau(t))$.

Remark 4: The proposed control law in (25) involves parameters $\gamma_{q}, K$ and $K_{1}$ which should be determined by designing in advance before it is implemented. Here, we can first determine the parameters $\gamma_{q}$ and matrix $\mathrm{K}$, for example $\gamma_{q}=1200$ and $K=0.4 I_{3}$, and then $\|P B K\|, \quad \max _{q_{0}+q^{T} q=1}\left\{\left\|\frac{1}{2}\left(J-\sigma^{T} \sigma\right) K\left(q_{0} I_{3}+q^{\times}\right) K\right\|\right\}+\left\|\sigma^{T} D \sigma K+\gamma_{q} I\right\|$ $\left\|\sigma^{T} E \quad \sigma^{T} D+P B\right\|+\max _{q_{0}+q^{T} q=1}\left\{\left\|(K q)^{\times} \sigma^{T}\right\|\right\}$ and $\begin{array}{r}\max _{q_{0}+q^{T} q=1}\left\{\left\|\frac{1}{2}\left(J-\sigma^{T} \sigma\right) K\left(q_{0} I_{3}+q^{\times}\right)\right\|\right\}-\left\|\sigma^{T} D \sigma\right\| \text { can be determined } \\ \hline\end{array}$ accordingly based on the constraint condition $\|q\|^{2}+q_{0}^{2}=1$. Finally, an appropriate gain matrix $\mathrm{K}^{1}$ can be selected to make the matrix inequality (19) satisfied. The given parameter $\gamma$ in (22) represents the disturbance attenuation level of the resulting closed system in (1), (12) and (16). As discussed in [26], any level of $L_{2}$ disturbance attenuation can be achieved by choosing $\gamma$ sufficiently small at the expense of a larger control effort.

From Theorem 1, we can obtain the amplitude constrained attitude controller (ACAC) for normal system in (1) and (4)-(6):

$$
u_{c}=2 \Sigma^{T}\left(\Sigma \Sigma^{T}\right)^{-1} \operatorname{SAT}_{M}\left(\frac{\tau}{2}, \frac{\tau_{\max }}{2}, x\right)
$$

with (25)-(28). Based on Theorem 1 and equation (35), the overall structure of the ACAC is shown in Figure 1, and the design procedure for ACAC is given as follows.

Step 1: Given $\gamma>0$ and select the parameter, $\gamma_{q}, \mathrm{~K}$ and $\mathrm{K}_{1}$ based on Remark 4 such that the matrix 
inequality in (19) is satisfied;

Step 2: Select compensator parameter diagonal matrix $\kappa$, set $G(0)=[0,0,0]^{T}$ and construct the saturation compensator 1 in (27)(28), where the input of the saturation compensator can be obtained by the feedback loop as shown in Figure 1;

Step 3: Obtain the controller u in (25);

Step4: Further get the ACAC controller $u_{c}$ based on (35).

The proposed amplitude constrained attitude controller in (35) with (26)-(28) achieves asymptotical stability of the resulting closed-loop attitude system with fault-free actuators. However, when actuator faults occur, it no longer ensures the stabilization and accuracy for the attitude control system. Therefore, to guarantee the stability of the system, a controller that can accommodate the actuator faults is needed for flexible spacecraft during actuator fault occurrence. In the follows, in order to propose the fault-tolerant constrained controllers, a significant Lemma is proposed.

Lemma 1: For the actuator distribution matrix $\Sigma$, fault switch matrix $\wedge$ and actuator effectiveness matrix $F$ under assumption 2, there exists a function matrix $S(t)$ and a constant $\bar{s}>0$, such that the following equation holds:

$$
\Sigma(I-\wedge(t)) F(t) \Sigma^{T}=S(t) \Sigma \Sigma^{T} \geq \bar{s} \Sigma \Sigma^{T}
$$

Proof: Because $\operatorname{rank}(\Sigma)=3$, therefore $\Sigma \Sigma^{T} \in R^{3 \times 3}$ is a positive definite symmetric matrix. Let $S(t)=\left(\Sigma(I-\wedge(t)) F(t) \Sigma^{T}\right)\left(\Sigma \Sigma^{T}\right)^{-1}$ , we have $S(t) \Sigma \Sigma^{T}=\Sigma(I-\wedge(t)) F(t) \Sigma^{T}$. As rank $\operatorname{rank}((I-\wedge(t)) F(t)) \geq 3,(I-\wedge(t)) F(t)=\operatorname{diag}\left(F_{1}, \ldots, 0\right)$, without loss of generality, suppose $(I-\wedge(t)) F(t)=\operatorname{diag}\left(F_{1}, . . \mathrm{F}_{L},, 0, \ldots, 0\right)$ with $F_{i}>0, i=1, \ldots, \mathrm{L}_{1}, \mathrm{~L}_{1} \geq 3$ and then there exists a constant $\bar{s}>0$ such that $F_{i}>0, i=1, \ldots, \mathrm{L}_{1}$ Let $\Sigma=\left[\Sigma_{1}, \Sigma_{2}\right]$ with $\Sigma_{1}$ and $\Sigma_{2}$ being $3 \times \mathrm{L}_{1}$ and $3 \times\left(\mathrm{L}-\mathrm{L}_{1}\right)$ orders, respectively, and then $\Sigma(I-\wedge(t)) F(t)=\left[\Sigma_{1}, \Sigma_{2}\right] \operatorname{diag}\left(F_{1}, \ldots F_{L}, 0, . .0\right)=\left[\Sigma_{1} \operatorname{diag}\left(F_{1}, \ldots F_{L}\right), 0_{3 \times\left(\mathrm{L}-\mathrm{L}_{1}\right)}\right)$. As

$X \Sigma((I-\wedge(t)) F(t)-\bar{S} I) \Sigma X^{T}=\left(X \Sigma_{1}\right)\left(\operatorname{diag}\left(F_{1}, \ldots F_{L}\right)-\bar{S} I\right)\left(X \Sigma_{1}\right)^{T}>0, \forall X \neq 0$, therefore we have $\operatorname{rank}\left(\Sigma_{1}\right)=3$ based on the fact $3 \geq \operatorname{rank}\left(\Sigma_{1}\right) \geq \min \left\{\operatorname{rank}\left(\Sigma_{1}\right), \operatorname{rank}\left(\Sigma_{1} \operatorname{diag}\left(F_{1}, \ldots F\right)\right)=3\right\}$.

Let $X \in R^{3}$ be a row vector. As $\operatorname{rank}\left(\Sigma_{1}\right)=3$ system of linear equations $X \Sigma_{1}=0$ only has zero solution. So, we have $X \Sigma_{1} \neq 0$ for $\forall X \neq 0$. As $X \Sigma((I-\wedge(t)) F(t)-\bar{s} I) \Sigma X^{T}=\left(X \Sigma_{1}\right)\left(\operatorname{diag}\left(F_{1}, \ldots F_{L}\right)-\bar{s} I\right)\left(X \Sigma_{1}\right)^{T}>0, \forall X \neq 0$, which implies that $\Sigma(F(t)(I-\wedge(t))-\bar{s} I) \Sigma^{T} \quad$ is a positive definite symmetric matrix. Therefore we have $(t) \Sigma \Sigma^{T}=\left(\Sigma(I-\wedge(t)) F(t) \Sigma^{T}\right) \geq \bar{s} \Sigma \Sigma^{T}$ this proof is completed.
As the fault switch matrix $\wedge$ and the actuator effectiveness matrix $\mathrm{F}$ are unknown, the constant $\bar{s}$ is also unknown, which needs to be estimated in the following control design. From Assumption 3 and the fact $\|\wedge\| \leq 1$ we have $\left\|\wedge u_{k h}\right\| \leq \bar{u}_{k h}(37)$

Based on Theorem 1 and Lemma 1, we propose the following fault-tolerant amplitude constrained attitude controller (FTACAC) in (38) with (39)-(44).

Theorem 2: Consider the flexible spacecraft system that consists of (1), (12) and (16) under actuator faults in (6) for which Assumptions 1-5 hold. Given $\gamma>0$, let $\gamma_{q}>0, \kappa=\operatorname{diag}\left(\kappa_{1}, \kappa_{2}, \kappa_{3}\right) \in R^{3 \times 3}$, where $\kappa_{i}>0,-1,2,3, \kappa \in R^{3 \times 3}$ and $\kappa_{1} \in R^{3 \times 3}$ be positive definite symmetric matrices. If $\gamma_{q}, \mathrm{~K}$ and $\kappa_{1}$ satisfy the inequality in (19), then the dynamic feedback control law

$$
u_{c}=-2 \lambda^{\max } \Sigma^{T} F_{2}^{-1} x=2 \lambda^{\max } \Sigma^{-1} \Sigma^{T} S A T_{M}\left(\frac{\tau}{2}, \frac{\tau_{\max }}{2}, x\right)
$$

Where

$\tau=\hat{\bar{s}} \psi+l$
$\psi=-\left[\frac{1}{\gamma_{q}}\left\|\omega^{\times}\left(J-\sigma^{T} \sigma\right)^{T}\left(K+\gamma_{q} K_{1}^{-1}\right)\left(\omega^{\times}\left(J-\sigma^{T} \sigma\right)\right)\right\|+2\left\|K_{1}\right\|+\frac{2}{\gamma^{2}}+2\left\|\Sigma \hat{u}_{k h}\right\|\right] x$

$$
\begin{gathered}
l=-\kappa G-\dot{G} \\
\dot{G}=-\kappa G+\left(2 \operatorname{SAT}_{M}\left(\frac{\tau}{2}, \frac{\tau_{\max }}{2}, x\right)-\tau\right) \\
\dot{\hat{s}}=\frac{1}{\gamma_{\bar{s}}^{2}}\|\psi\|\|x\|-\frac{\beta_{\bar{s}}}{\gamma_{\bar{s}}^{2}}\|x\| \hat{\bar{s}} \\
\dot{\hat{\bar{u}}}_{k h}=\frac{1}{\gamma_{\bar{u}_{k h}}^{2}}\|x\|-\frac{\beta_{\bar{u}_{k h}}}{\gamma_{\bar{u}_{k h}}^{2}}\|x\| \hat{\bar{u}}_{k h}
\end{gathered}
$$

$\lambda^{\max }$ Denotes the maximum singular value of matrix $\left(\Sigma \Sigma^{T}\right)^{-1}$

$\left(\Sigma \Sigma^{T}\right)^{-1}, \hat{\bar{s}}, \hat{\bar{u}}_{k h}$ are the estimates of $1 / \bar{s}$ and $\hat{\bar{u}}_{k h}$ respectively, $\gamma_{\bar{s}}, \beta_{\bar{s}}, \gamma_{\bar{u}_{k h}}$ and $\beta_{\bar{u}_{k h}}$ re positive constants, renders the closed loop system in (1), (12) and (16) under actuator faults in (6) ISS from the input $\left[d^{T}, \bar{s}, \hat{\bar{u}}_{k h}\right]^{T}$ to the state $\left[q^{T} x^{T}, \xi^{T}\right]^{T}$

Proof: Define the estimation errors of $1 / \bar{s}$ and $\hat{\bar{u}}_{k h}$ as follows: $\tilde{\bar{s}}=\hat{\bar{s}}-\frac{1}{\bar{s}}$ and $\tilde{\bar{u}}_{k h}=\bar{u}_{k h}-\hat{\bar{u}}_{k h}$. Following the same steps as the proof in Theorem 1, we consider the smooth positive-definite radically unbounded function as follows

$V_{1}=\frac{1}{2} \gamma q^{T} q+\frac{1}{2} \gamma_{q}\left(1-q_{0}\right)^{2}+\frac{1}{2} x^{T}\left(J-\sigma^{T} \sigma\right) x+\frac{1}{2} \xi^{T} P \xi+\frac{1}{2} \gamma_{\bar{s}}^{2} \tilde{s}^{2}+\frac{1}{2} \gamma_{\bar{u}_{k h}}^{2}\left\|\sum\right\| \tilde{\bar{u}}_{k h}^{2}$

The time derivate $\mathrm{V}_{1}$ along (1), (6), (12) and (16) is given by 


$$
\begin{aligned}
& \dot{V}_{1}=-\gamma_{q} q^{T} K q+\gamma_{q} x^{T} q+\xi^{T} P(A \xi+B \omega)+\gamma_{\bar{s}}^{2} \frac{\tilde{s} \hat{s}}{s}-\gamma_{\bar{u}_{k h}}^{2}\|\Sigma\| \tilde{\tilde{u}}_{k h}+ \\
& x^{T}\left[-\omega^{\times}\left(J-\sigma^{T} \sigma\right) \omega-\sigma^{T} D \sigma \omega+\frac{1}{2}\left(J-\sigma^{T} \sigma\right) K\left(q_{0} I_{3}+q^{\times}\right) \omega+\left[\begin{array}{ll}
\sigma^{T} E & \left.\left.\sigma^{T} D-\omega^{\times} \sigma^{T}\right] \xi+\Sigma\left(\wedge u_{k h}+(I-\wedge) F u_{c}(t)\right)+d\right]
\end{array}\right.\right.
\end{aligned}
$$

From update law in (43), we can obtain that $\hat{\bar{s}}>0$ if choosing $\hat{\bar{s}}(0)>0$. Therefore, we have the following

Inequality hold based on Lemma 1:

$-x^{T} \Sigma(I-\wedge(t)) F(t) \Sigma^{T} \lambda^{\max } F^{-1} x \leq 2 \overline{s x}^{T} S A T T_{M}\left(\frac{\tau}{2}, \frac{\tau_{\max }}{2}, x\right) \leq \overline{s x}^{T} \operatorname{SAT} T_{M}\left(\frac{\tau}{2}, \frac{\tau_{\max }}{2}, x\right)$

The last inequality in (47) is based on the fact that $\hat{S S X}^{T} S A T\left(\tau, \tau_{\max }, x\right) \leq 0$. In order to prove $\dot{V}_{1}<0$, we first prove the following inequality holds

$x^{T} \Sigma\left(\wedge u_{k h}+(I-\wedge) F u_{c}(t)\right)+\gamma_{\bar{u}_{k h}}^{2} \frac{\tilde{\dot{S}}}{S S \gamma_{\bar{u}_{k h}}^{2}}\|\Sigma\| \tilde{\bar{u}}_{k h} \dot{\hat{\bar{u}}}_{k h}$

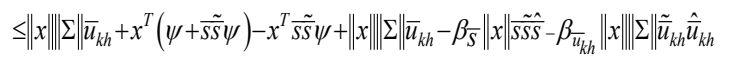

$=x^{T} \psi+\|x\|\|\Sigma\| \hat{\bar{u}}_{k h}-\beta_{\bar{s}}\|x\| \bar{s}\left(\tilde{\bar{s}}+\frac{\bar{s}}{2}\right)^{2}+\frac{1}{4} \beta_{\bar{s}}\|x\| \overline{s s}^{2}-\beta_{\bar{u}_{k h}}\left\|\sum\right\| x\left\|\left(\tilde{\tilde{u}}_{k h}+\frac{\bar{u}_{k h}}{2}\right)^{2}+\beta_{\bar{u}_{k h}}\right\| x\|\| \Sigma \| \tilde{\bar{u}}_{k h} \hat{\bar{u}}_{k h}$

So from Theorem 1 and (46)-(48) we can obtain

$\dot{V}_{1} \leq-\lambda_{\wedge}^{\min }\left(\|q\|^{2}+\|x\|^{2}+\|\xi\|^{2}\right)+\|x\|\left(\|d\|+\frac{1}{4} \beta_{\bar{S}} \bar{S}^{3}+\frac{1}{4} \beta_{\bar{u}_{k h}}\|\Sigma\| \bar{u}_{k h}^{2}-\|x\| / \gamma^{2}\right)$

Choosing

$\rho\left(\left|\left[d^{T}, \bar{S}, \bar{u}_{k h}\right]^{T}\right|\right)=\gamma^{2}\left(\|d\|+\frac{1}{4} \beta_{\bar{S}} \bar{S}^{3}+\beta_{\bar{u}_{k h}}\|\Sigma\| \bar{u}_{k h}^{2}\right) \in \mathrm{K}_{\infty}, \quad$ then

when $\|x\| \geq \gamma^{2}\left(\|d\|+\frac{1}{4} \beta_{\bar{S}} \bar{S}^{3}+\beta_{\bar{u}_{k h}}\|\Sigma\| \bar{u}_{k h}^{2}\right)$ we have $\dot{V}_{1}=0$, which implies that $\mathrm{V}_{1}$ in (45) is ISS-CLF based on Definition 1. Therefore, the resulting closed loop system in (1), (12) and (16) under actuator faults in (6) is ISS from the input $\left[d^{T}, \bar{S}, \bar{u}_{k h}\right]^{T}$ to the state $\left[q^{T}, x^{T}, \xi^{T}\right]^{T}$. The proof of Theorem 2 is completed.

Remark 5: The second terms of parameter update laws in (43)(44) are the e-modifications, which guarantee bounded parameter estimates. Based on Theorem 2, the overall structure of the FTACAC is shown in Figure 2, and the design procedure for FTACAC is given as follows

Step1: Given $\gamma>0$ and select the parameter $\gamma_{q}, \mathrm{~K}$ and $\mathrm{K}_{1}$ based on Remark 4 such that the matrix inequality in (19) is satisfied;

Step 2: Select compensator parameter diagonal matrix $\kappa$, set $G(0)=[0,0,0]^{T}$ and construct the saturation compensator 1 in (41)(42), where the input $(u-\tau)$ of the saturation compensator can be obtained by the feedback loop as shown in Figure 2;

Step 3: Select parameters, $\gamma_{s}, \beta_{s}$ and $\gamma_{\bar{u}_{k h}}$, set $\hat{\bar{s}}(0)=1$ and $\bar{u}_{k h}(0)=0.5$, and construct the parameters update laws in (43)-(44);
Step4: Obtain the FTACAC controller $u_{c}$ in (38).

A fault-tolerant amplitude and rate constrained attitude controller design: Control law in (38) only accounts for actuator amplitude constraint. In fact, many cases we require not only the amplitude constraint but also rate constraint of actuators to limit the possible excitation of high-frequency UN modeled dynamic of spacecraft as discussed in Wallsgrove RJ ${ }^{9}$. However, there are still few results that take both actuator amplitude and rate constraints into account in the attitude control of spacecraft. To account for the amplitude and rate constraints, we proposed the fault-tolerant amplitude and rate constrained attitude controller (FTARCAC) in (50) with (51)-(58) based on the following Theorem 3.

Theorem 3: Consider the flexible spacecraft system that consists of (1), (12) and (16) under actuator faults in (6) for which Assumptions $1-5$ hold, given $\gamma>0$ let $\gamma_{q}>0, \quad \kappa=\operatorname{diag}\left(\kappa_{1}, \kappa_{2}, \kappa_{3}\right) \in R^{3 \times 3}$, where $\kappa_{i}>0,-1,2,3, \kappa \in R^{3 \times 3}$ and $\kappa_{1} \in R^{3 \times 3}$ be positive definite symmetric matrices. If $\gamma_{q}, \mathrm{~K}$ and $\mathrm{K}_{1}$ satisfy the inequality in (19), then the dynamic feedback control law

$u_{c}=-2 \lambda^{\max } \sum_{\left(\Sigma \Sigma^{T}\right)^{-1} \Sigma^{T} F_{3}^{-1} x=2 \lambda^{\max }}\left(\Sigma \Sigma^{T}\right)^{-1} \Sigma^{T} S A T\left(\frac{\tau}{2}, \frac{\tau^{\#}}{2}, \mathrm{Rax}, x\right)=\lambda_{\left(\Sigma \Sigma^{T}\right)^{-1} \Sigma^{T} u}^{\max }$

With the time derivative of $u=2 S A T\left(\frac{\tau}{2}, \frac{\tau_{\text {max }}^{\#}}{2}, \mathrm{R}, x\right)$ defined as

$$
\dot{u}=S_{R} T_{R}\left(\bar{\omega}\left(2 \operatorname{SAT}_{M}\left(\frac{\tau}{2}, \frac{\tau_{\text {max }}^{\#}}{2}, \mathrm{R}, x\right)-u\right)\right)
$$

Where $\bar{\omega}=\operatorname{diag}\left(\bar{\omega}_{1}, \bar{\omega}_{2}, \bar{\omega}_{3}\right) \in R^{3}$,

$$
\tau=\hat{\bar{s}} \psi+l
$$

$\psi=-\left[\frac{1}{\gamma_{q} \|}\left\|\left(\omega^{\times}\left(J-\sigma^{T} \sigma\right)\right)^{T}\left(K+\gamma_{q} K_{1}^{-1}\right)\left(\omega^{\times}\left(J-\sigma^{T} \sigma\right)\right)\right\|+2\left\|k_{1}\right\|+\frac{2}{\gamma^{2}}+2\left\|\sum \hat{u}_{k h}\right\|\right] x$

$$
\begin{gathered}
l=-\kappa G-\dot{G} \\
\dot{G}=\kappa G+\left(2 S A T \left(\frac{\tau}{2}, \frac{\tau_{\text {max }}^{\#}}{2}, \mathrm{R}, x\right.\right. \\
\tau_{\text {max }}^{\#}=\tau_{\text {max }}-\|R\| /\|\omega\|>0 \\
\dot{\hat{\bar{s}}}=\frac{1}{\gamma^{\frac{2}{s}}}\|\psi\|\|x\|-\frac{\beta_{\bar{s}}}{\gamma^{\frac{2}{s}}}\|x\| \hat{\bar{s}} \\
\dot{\hat{\bar{u}}}_{k h}=\frac{1}{\gamma_{\bar{s}}^{2}}\|x\|-\frac{\beta_{\bar{u}_{k h}}}{\gamma_{\bar{u}_{k h}}^{2}}\|x\| \hat{\bar{u}}_{k h}
\end{gathered}
$$$$
\dot{G}=\kappa G+\left(2 \operatorname{SAT}\left(\frac{\tau}{2}, \frac{\tau_{\text {max }}^{\#}}{2}, \mathrm{R}, x\right)-\tau\right)
$$ 
Renders the closed loop system in (1), (12) and (16) under actuator faults in (6) ISS from the input $\left[d^{T}, \bar{S}, \bar{u}_{k h}\right]^{T}$ to the state $\left[q^{T}, x^{T}, \xi^{T}\right]^{T}$.

Proof: following Theorem 1 and Theorem 2, the proof can be completed and therefore we omit it. Finally, we prove control law u in (50) satisfying $\|u\| \leq \tau_{\text {max }}$ Define $\varepsilon(x)=u-2 S A T_{M}\left(\frac{\tau}{2}, \frac{\tau^{\#}{ }_{\text {max }}}{2}, x\right)$ From (51) and the and the $S A T_{R}$ in (18), we can obtain $\|\varepsilon(x)\| \leq\|R\| /\|\bar{\omega}\|$. Therefore $\|u\| \leq\left\|\tau_{\text {max }}^{\#}\right\|+\|\varepsilon(x)\| \leq \tau_{\text {max }}$ So the inequality in (8) is satisfied.

Remark 6: The overall structure of the FTARCAC is shown in Figure 3. Equation (51) can be considered as a linear, stable and lowpass filter, where $\bar{\omega}=\operatorname{diag}\left(\bar{\omega}_{1}, \bar{\omega}_{2}, \bar{\omega}_{3}\right)$ is the natural frequency of the filter. By employing the filter in (51), the robust controller in (50) with (51)-(58) accounts for not only amplitude constraint but also rate constraint, as well as actuator faults in the presence of inertia matrix uncertainty and external disturbance.

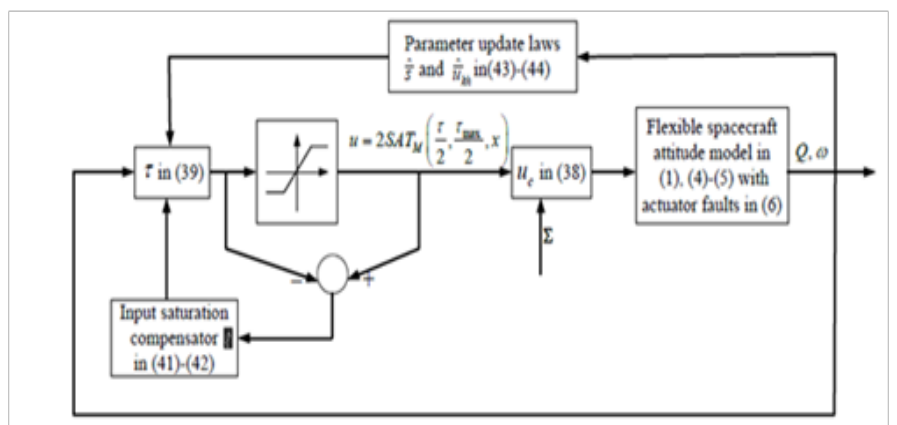

Figure I The overall structure of FTACAC.

\section{Numerical simulations}

In this section, numerical simulations are presented to demonstrate the effectiveness of the proposed ACAC in (25) with (26)-(28), FTACAC in (38) with (39)-(44) and FTARCAC in (50) with (51)(58). The anti-windup Proportional-integral-derivative (AWPID) method in Bang ${ }^{11}$ is also performed for the purpose of comparison. The rest-to-rest maneuver of the flexible spacecraft is considered in the following simulations. Parameters used in all simulations are given as follows. The nominal value component of the inertia matrix. $\mathrm{J}_{\mathrm{s}}$ for a spacecraft is described as ${ }^{22}: J=\left[\begin{array}{ccc}350 & 3 & 4 \\ 3 & 280 & 10 \\ 4 & 10 & 190\end{array}\right] \mathrm{kg} / \mathrm{m}^{2}$ ,and the perturbation component is given by $\Delta J=\left[\begin{array}{ccc}4.2+0.5 \cos t & 3 & 4+\sin 5 t \\ 3 & -7+\cos 3 t & 2.5 \\ 4+0.1 \sin 5 t & 2.5 & 5.89+1.5 \sin t\end{array}\right] \mathrm{kg} / \mathrm{m}^{2} ;$ the couple matrix is $\sigma=\left[\begin{array}{ccc}6.45637 & 1.27814 & 2.15629 \\ -1.25619 & 0.91756 & -1.67264 \\ 1.23637 & -2.6581 & -1.12503\end{array}\right]$;

The first four elastic modes are considered and, the damping and stiffness matrices of the flexible appendages are given by
$D=\left[\begin{array}{cccc}0.0086 & 0 & 0 & 0 \\ 0 & 0.19 . & 0 & 0 \\ 0 & 0 & 0.0487 & 0 \\ 0 & 0 & 0 & 0.1275\end{array}\right]$ and

$E=\left[\begin{array}{cccc}0.3900 & 0 & 0 & 0 \\ 0 & 1.2184 & 0 & 0 \\ 0 & 0 & 3.5093 & 0 \\ 0 & 0 & 0 & 6.5005\end{array}\right]$

respectively; To

consider possible spillover effects, only the first three elastic modes are

taken into account in the controller design; The actuators distribution matrix is given by $\mathrm{Hu}^{22} \Sigma=\left[\begin{array}{cccc}\sqrt{3} / 3 & -\sqrt{3} / 3 & -\sqrt{3} / 3 & \sqrt{3} / 3 \\ -\sqrt{3} / 3 & -\sqrt{3} / 3 & -\sqrt{3} / 3 & -\sqrt{3} / 3 \\ \sqrt{3} / 3 & \sqrt{3} / 3 & -\sqrt{3} / 3 & -\sqrt{3} / 3\end{array}\right]$ that is to say, four actuators are considered in the following simulations; The disturbance toque is given by $d_{s}(t)=\left(\|\omega\|^{2}+0.15\right)\left[\begin{array}{lll}\cos (0.1 t) & \sin (0.5 t) & \sin (0.3 t)\end{array}\right]^{T} \quad$ The maximum allowable torque input is $\tau_{\max }=10 \mathrm{~N} . \mathrm{m}$ The initial attitude orientation of the unit quaternion is $q(0)=\left[\begin{array}{lll}-0.2 & 0.7 & -0.35\end{array}\right]^{T}$ and the initial angular velocity is $\omega(0)=\left[\begin{array}{lll}0 & 0 & 0\end{array}\right]^{T}$ the initial vibration state of flexible appendage is $\eta_{i}(0)=\dot{\eta}_{i}(0)=0, i=1,2,3,4$ The initial value of the adaptive parameters are $\hat{\bar{s}}(0)=1$ and $\hat{\bar{u}}_{k h}(0)=0.5$ The control gains of the proposed controllers are given by $r_{q}=1200, K=0.4 I, K_{1}=700 I, \gamma=2, K=\operatorname{diag}(2,2,2), \gamma_{\bar{s}}=\sqrt{5}, \beta_{\bar{s}}=0.5, \gamma_{\overline{u_{k h}}}=0.5, \bar{\omega}=\operatorname{diag}(5,5,5)$ and $R=[10,10,10]^{T}$. The control gains of the AWPID are designed after repeated attempts until good control performances are obtained. Two cases of actuator scenario are considered in the following simulations, i.e., all actuators are normal and some actuators occur faults at some moments. In the actuator fault scenario, we consider that one actuator undergoes the partial loss of effectiveness during $5 s \leq t \leq 70 s$ that is $F_{1}=\left\{\begin{array}{cl}0.5+0.05(\cos (2 \pi t)-1) & 5 s \leq t \leq 70 s \\ 1 & \text { otherwise }\end{array}\right.$, and another experiences stuck fault at $t \geq 10 \mathrm{~s}$ given by $u_{k h 2}=0.45+0.1 \sin t \quad t \geq 10 \mathrm{~s}$. The others are normal in the whole process of the spacecraft operator.

\section{Faulty case}

In this section, we consider the case that some actuators generate the partial loss of effectiveness and stuck fault at some moments in the process of attitude control for flexible spacecraft. Figures 2-8 show the simulation results of the FTACAC, FTARCAC and AWPID. Figure 2, Figure 3 show the trajectories of the unit quaternion and angular velocity of the flexible spacecraft under the three controllers, which can be seen that, when actuator faults occur, acceptable control 
performance and convergence rate of the resulting closed-loop system can still be obtained by FTACAC and FTARCAC in the presence of input saturation, inertia matrix uncertainty and external disturbance. While for AWPID, unexpected control result happens under actuator faults occurrence. The reason is that when actuators of spacecraft undergo faults especially severe faults, the stability of the control system can no longer be guaranteed by the conventional controller such as AWPID. While for our proposed FTACAC and FTARCAC, as we employ two adaptive parameters $\hat{\bar{s}}$ and $\hat{\bar{u}}_{k h}$ eliminate the effect of the actuator faults in the controller design, therefore acceptable control performance can also be achieved even though severe actuator faults occur. Figure 4 shows the trajectories of the flexible vibration, from which we can see that, a serious vibration of flexible appendages appears for AWPID. While for FTACAC and FTARCAC, a low vibration level also achieved. Figure 5, Figure 6 show the trajectories of the thruster outputs and compensator 1. Figure 7, Figure 8 show the trajectories of adaptive parameters $\hat{\bar{s}}$ and $\hat{\bar{u}}_{k h}$ from which we can find that, the estimation parameters $\hat{\bar{s}}$ and $\hat{\bar{u}}_{k h}$ are convergent but uncertain convergent to the true parameters $1 / \bar{s}$ and $\bar{u}_{k h}$, respectively.

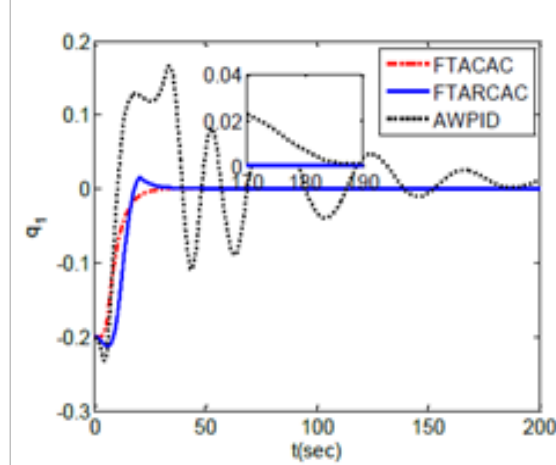

(a)



(b)

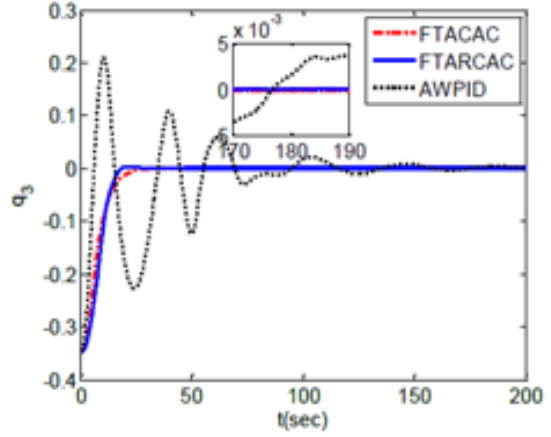

(c)

Figure 2 Quaternion with faulty actuators.

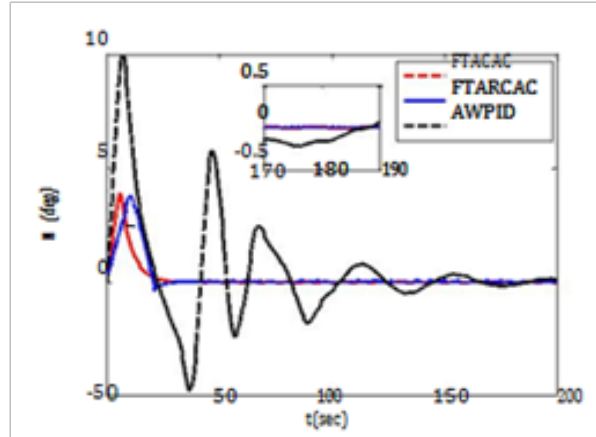

(a)

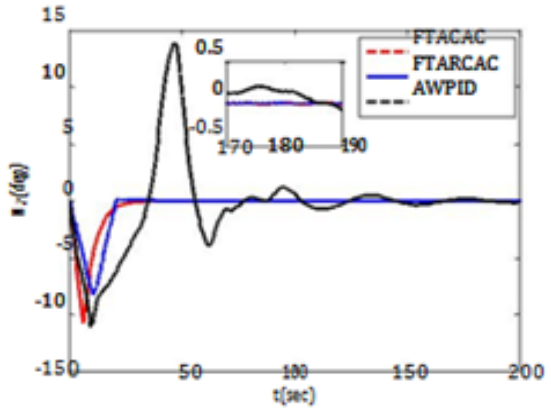

(b)

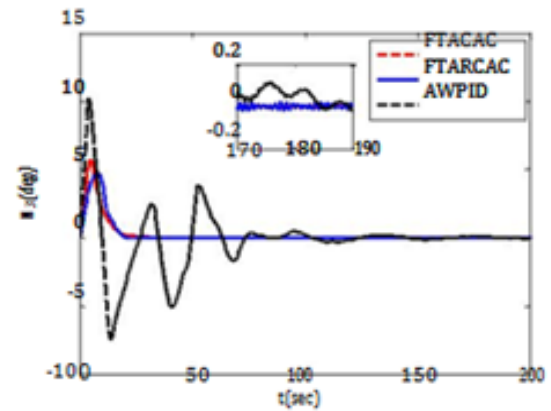

(c)

Figure 3 Angular velocity with fault actuators.

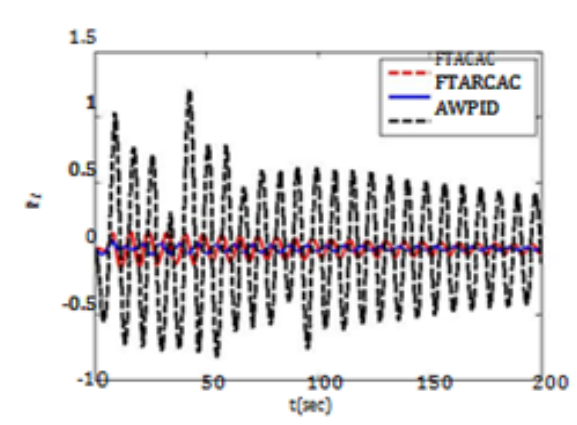

(a)

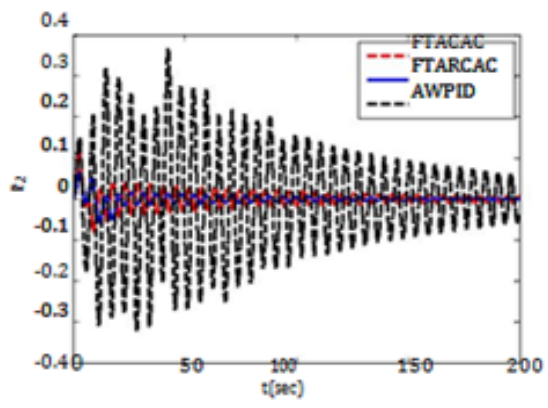

(b)

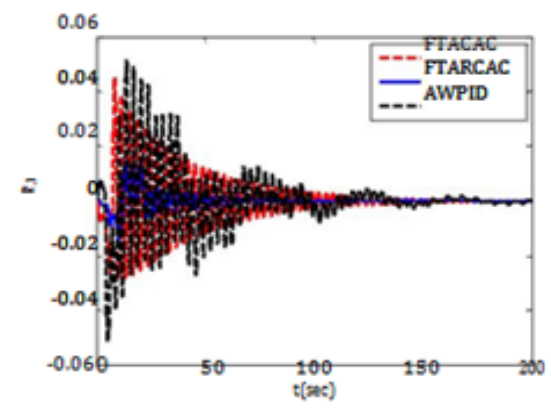

(c)

Figure 4 Flexible vibration with fault actuators. 


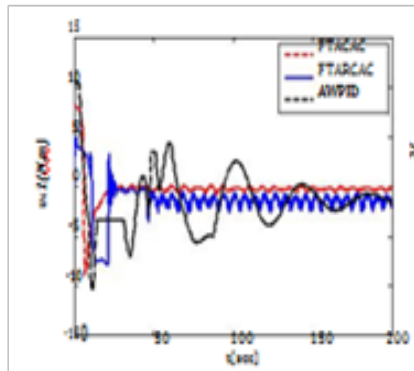

(a)



(b)

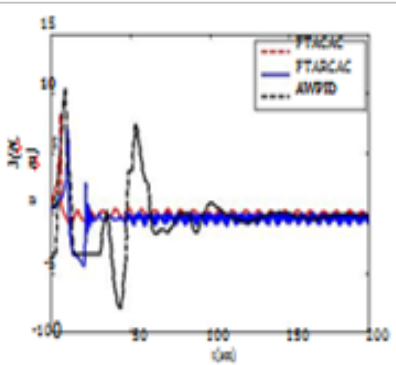

(c)

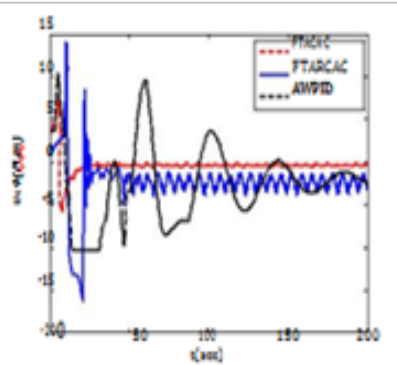

(d)

Figure 5 Thruster outputs with fault actuators.

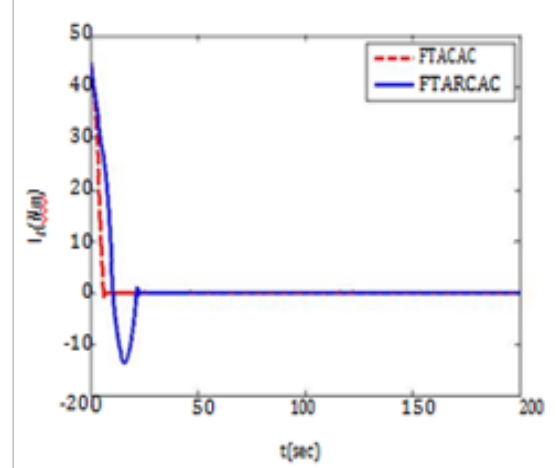

(a)

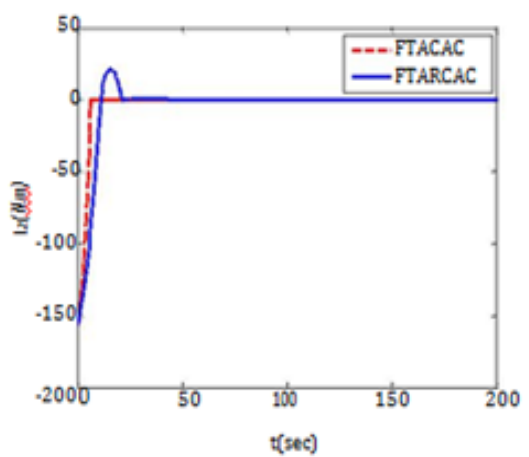

(b)

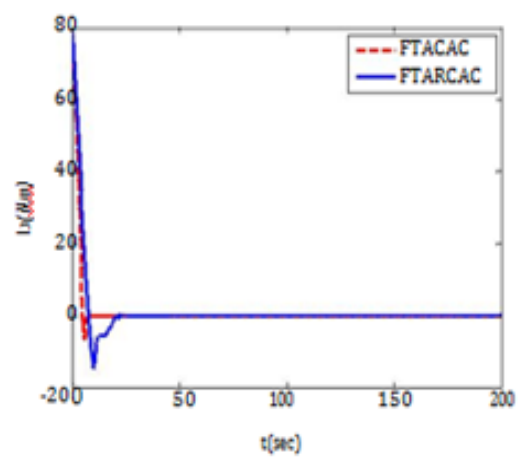

(c)

Figure 6 Compensator with fault actuators.

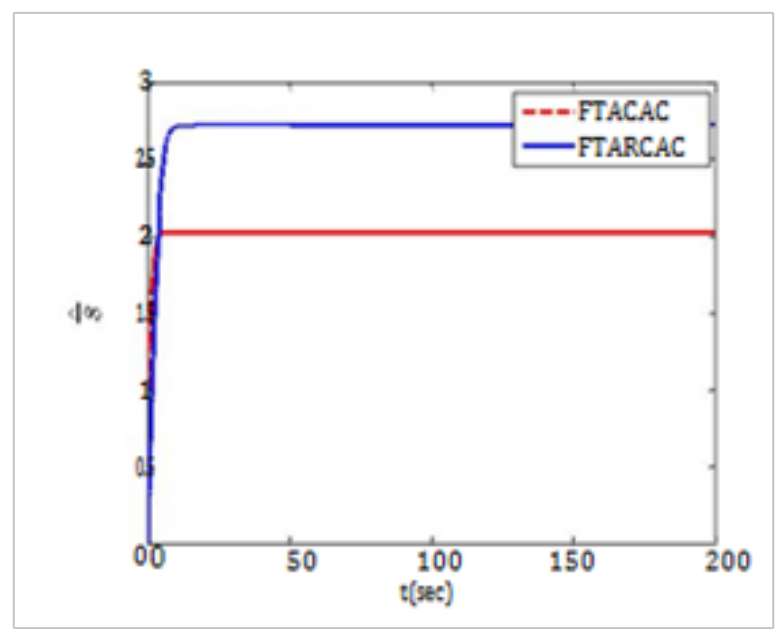

Figure7 Estimation parameter.

\section{Conclusion}

This paper presents robust constrained fault-tolerant attitude control algorithms for flexible spacecraft in the presence of actuator fault, control input saturation, model uncertainty and external disturbances. In order to compensate the effect of input saturation, a compensator is employed in the controller design. To handle the actuator amplitude and/or rate constraints under actuator fault

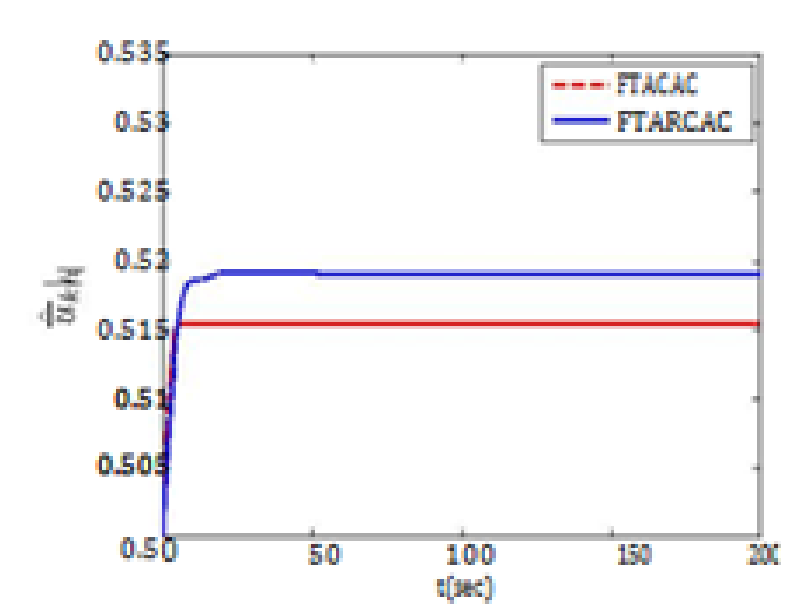

Figure 8 Estimation parameter.

occurrence, two constrained fault-tolerant controllers are designed by two parameter update laws to estimate the unknown parameters caused by actuator faults. The proposed controllers are assessed and compared with AWPID through numerical simulations. The result shows that the proposed constrained fault-tolerant attitude controllers are able to accommodate the actuator fault and achieve high precision pointing while conventional methods fail to attain expected control objective. 


\section{Acknowledgments}

None.

\section{Conflict of interest}

Author declares that there are none of the conflicts.

\section{References}

1. Jasim NF, jasim IF. Robust adaptive control of spacecraft attitude systems with unknown dead zones of unknown bounds. J Syst Control Eng. 2012;226(7):947-955.

2. Song Z, Li H, Sun K. Finite-time control for nonlinear spacecraft attitude based terminal sliding mode technique. ISA Trans. 2014;53(1):117-124.

3. Wu S, Radice G, Gao Y, et al. Quaternion-based finite time control for spacecraft attitude tracking. Acta Astronautica. 2011;69(1-2):48-58.

4. Nguyen T, Jabbari F. Output feedback controllers for disturbance attenuation with actuator amplitude and rate saturation. Automatica. 2000;36(9):1339-1346.

5. Leonessa A, Haddad WM, Hayakawa T, et al. Adaptive control for nonlinear uncertain systems with actuator amplitude and rate saturation constraints. Int J Adapt Control signal process. 2009;23(1):73-96.

6. Yuan R, Tan X, Fan G, et al. Robust adaptive neural network control for a class of uncertain nonlinear systems with actuator amplitude and rate saturations. Neurocomputing. 2013;125:72-80.

7. Boškovié JD, Li SM, Mehra RK, et al. Robust adaptive variable structure control of spacecraft under control input saturation. J Guid Control Dyn 2001;24(1):14-22.

8. Boškovié JD, SM Li, Mehra RK. Robust tracking control design for spacecraft under control input saturation. J Guid Control Dyn. 2004;27(4):627-633.

9. Wallsgrove RJ, Akella MR. Globally stabilizing saturated attitude control in the presence of bounded unknown disturbances. J Guid Control Dyn. 2005;28(5):957-963.

10. Zhu Z, Xia Y, Fu M. Adaptive sliding mode control for attitude stabilization with actuator saturation. IEEE Trans Indust Electronics. 2011;58(10):4898-4907.

11. Bang H, Tahk MJ, Choi HD. Large angle attitude control of spacecraft with actuator saturation. Control Eng Pract. 2003;11(9):989-997.

12. $\mathrm{Hu} \mathrm{QL}, \mathrm{Xiao} \mathrm{B}$. Intelligent proportional-derivative control for flexible spacecraft attitude stabilization with unknown input saturation. Aeros $\mathrm{Sci}$ tech. 2012;23(1):63-74.

13. Q L Hu, Xiao B. Robust adaptive back stepping attitude stabilization and vibration reduction of flexible spacecraft subject to actuator saturation. $J$ Vibrat Control. 2010;17(1):1657-1671.
14. Wang T, Xie W, Zhang Y. Sliding mode fault tolerant control dealing with modeling uncertainties and actuator faults. ISA Transactions. 2012;51(3):386-392.

15. Li XJ, Yang GH. Robust adaptive fault-tolerant control for uncertain linear systems with actuator failures. IET Control Theory Appl. 2012;6(10):1544-1551.

16. Jin JH, Ko SH, Ryoo CK. Fault tolerant control for satellites with four reaction wheels. Control Eng Pract. 2008;16(10):1250-1258.

17. Cai WC, Liao XH, Song YD. Indirect robust adaptive fault-toleran control for attitude tracking of spacecraft. J Guid Control Dyn. 2008;31(5):1456-1463.

18. Mirshams M, Khosrojerdi M, Hasani M. Passive fault-tolerant sliding mode attitude control for flexible spacecraft with faulty thrusters. $J$ Aeros Eng. 2013;228(12):1-15.

19. Lee H, Kim Y. Fault-tolerant control scheme for satellite attitude control system. IET control Theory Appl. 2009;4(8):1436-1450.

20. Meng Q, Zhang T, Song JY. Modified model-based fault-tolerant timevarying attitude tracking control of uncertain flexible satellites. $J$ Aerosp Control. 2012;227(11):1-15.

21. Jiang $\mathrm{Y}, \mathrm{Hu} \mathrm{Q}, \mathrm{Ma} \mathrm{G}$. Adaptive back stepping fault-tolerant control for flexible spacecraft with unknown bounded disturbances and actuator failures. ISA Trans. 2010;49(1):57-69.

22. $\mathrm{Hu} \mathrm{QL}$. Robust adaptive sliding-mode fault-tolerant control with wheels. IET control Theory Appl. 2009;4(6):1055-1070.

23. $\mathrm{Hu}$ QL. Adaptive integral-type sliding mode control for spacecraft attitude maneuvering under actuator stuck failures. Chinese J Aerona. 2011;24(1):32-45.

24. Krstic M. Inverse optimal stabilization of a rigid spacecraft. IEEE Trans Auto Control. 1999;44:1042-1049.

25. Luo WC, Chu YC, Ling KV. H infinity inverse optimal attitude-tracking control of rigid spacecraft. J Guid Control Dyn. 2005;28(3):481-493.

26. Luo WC, Chu YC, Ling $\mathrm{K}$. Inverse optimal adaptive control for attitude tracking of spacecraft. IEEE Trans Auto Control. 2005;50(11):16391654.

27. Krstic M, Li ZH. Inverse optimal design of Input-to-State stabilizing nonlinear controllers. IEEE Trans Auto Control. 1998;43(3):336-350.

28. M Krstic, Kanellakopoulos I, Kokotovic P. Nonlinear and adaptive control design. IEEE Trans Automatic Control. 1995;41(12):1-5. 